\title{
Evaluation of Arterial Wall Imaging with 16-slice Multi-detector Computed Tomography
}

\author{
RC Chan*, M Ferencik*, T Wu, U Hoffmann, TJ Brady, S Achenbach \\ Cardiac MR \& CT Program, Department of Radiology, Massachusetts General Hospital, \\ Harvard Medical School, Boston, MA, USA
}

\begin{abstract}
We have characterized the performance of 16-slice cardiac MDCT for assessment of coronary disease burden and lesion composition under optimal conditions. Morphology measurements with MDCT were accurate and reproducible. Detection of lipid-containing regions was possible with sensitivities $\geq 88 \%$ and specificity of $96 \%$ for regions $\geq 1.5 \mathrm{~mm}$ in size with $\geq 30 \%$ lipid. Estimation of lipid pool dimension is difficult and lipid pool CT densities cannot be used directly to determine the \%lipid content due to noise and blurring. These results represent the best-case performance of MDCT and help to guide interpretation of images obtained in vivo.
\end{abstract}

\section{Introduction}

Coronary atherosclerosis remains the major cause of mortality and morbidity in industrialized nations. Histopathology studies demonstrate that that majority of acute coronary events may be triggered by the rupture of certain high-risk or "vulnerable" plaques characterized by the presence of a thin fibrous cap overlying a large lipid pool or necrotic core. These lipid-rich regions are typically smaller than $5.0 \mathrm{~mm}^{2}$ and an estimated $80 \%$ of plaques have cores larger than $1.0 \mathrm{~mm}^{2}[1,2]$. The only clinical method currently available for assessment of coronary plaques in vivo is intravascular ultrasound (IVUS) and its invasiveness limits its use to patients who undergo coronary angiography. A non-invasive, rapid method for highresolution coronary wall imaging is required for screening and monitoring of disease burden in "high-risk" patients for whom catheterization is not justified.

Advances in multidetector spiral computed tomography (MDCT) now allow for simultaneous acquisition of projection data from 16 parallel slices with a spatial resolution of $0.6 \times 0.6 \times 0.75 \mathrm{~mm}$. Gantry rotation times of $420 \mathrm{~ms}$ allow for contrast-enhanced cardiac imaging with a temporal resolution of $\sim 210 \mathrm{~ms}$ and require only $\sim 20 \mathrm{~s}$ for complete coverage of typical hearts. An example of coronary wall imaging with 16-slice MDCT is illustrated in Figure 1, along with the corresponding IVUS image.
Recent reports on 16-slice cardiac MDCT demonstrate sensitivities between $93-95 \%$ and specificities between 86$92 \%$ for detection of significant coronary stenoses [3, 4]. In addition, CT densities reported in the literature for lipid-rich, fibrous, and calcific plaques fall roughly in the range of $14 \pm 24 \mathrm{HU}, 91 \pm 21 \mathrm{HU}$, and $419 \pm 194 \mathrm{HU}$, respectively $[5,6]$. These observations raise the possibility of characterizing lesion composition with 16-slice MDCT.

A key step towards the use of MDCT for coronary wall imaging is the systematic characterization of its performance when characterizing disease burden. Towards this goal, we determine the accuracy and reproducibility of 16-slice MDCT measurements of vessel morphology relative to those from IVUS and from "gross sections". We then focus on the ability of 16-slice MDCT to detect and characterize lipid-rich regions in non-calcified plaque to determine how reliably "vulnerable" lesions can be identified as a function of lipid pool size and composition. In the experiments presented here, we have used phantoms for the vessel wall and coronary lesions since they allow for greater control over morphological dimensions and lesion composition than is possible with arterial specimens.

\section{Methods}

\section{Vessel morphology phantoms}

Phantoms for the study of vessel morphology were constructed by injecting a molten polyvinyl alcohol (PVA) hydrogel (Lentikat $^{\circledR}$, geniaLab BioTechnologie, Braunschweig, Germany) into stainless steel cylindrical molds ranging from $1.7-3.1 \mathrm{~mm}$ in inner diameter and from

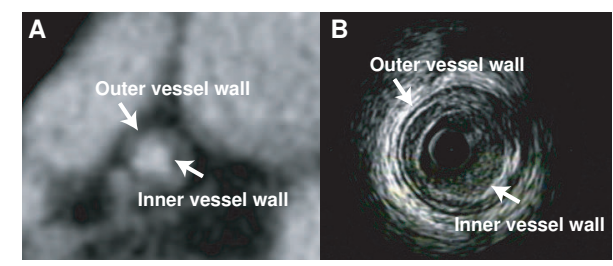

Figure 1. A. Multiplanar reformatted MDCT image of a coronary plaque in cross-section and $\mathbf{B}$. corresponding IVUS cross-section. 
3.0-4.0mm in outer diameter. PVA gelation was induced by freezing at $-20^{\circ} \mathrm{C}$ for $10 \mathrm{hrs}$ and then thawing for $10 \mathrm{hrs}$ at $4^{\circ} \mathrm{C}$. PVA lesions ranging in diameter from $0.6-1.5 \mathrm{~mm}$ were also constructed and attached to the PVA vessels to mimic diseased vessels with wall thicknesses varying between 0.7 $3.0 \mathrm{~mm}$. The CT density of the resulting PVA arteries was $60-95 \mathrm{HU}$, falling within the range typical of fibrous tissue. Morphology phantoms were immersed in oil to simulate the effect of perivascular fat and iodinated contrast (Ultravist$370^{\circledR}$, Schering, Berlin, Germany) was injected into the lumen to achieve enhancement typical of in vivo MDCT.

\section{Lesion composition phantoms}

To study the detectability of lipid-rich regions within fibrous tissue, a lucite block with CT densities of $\sim 98 \pm 25 \mathrm{HU}$ was used to simulate the HU mean and standard deviation observed in fibrous tissue. Cylindrical cavities $0.4-4.0 \mathrm{~mm}$ in diameter were drilled into the lucite block and fat emulsions were injected to introduce lipidrich regions of varying size. The lipid concentration of the injected emulsion was varied between imaging sessions to study the effect of lesion composition on lipid-pool detectability.
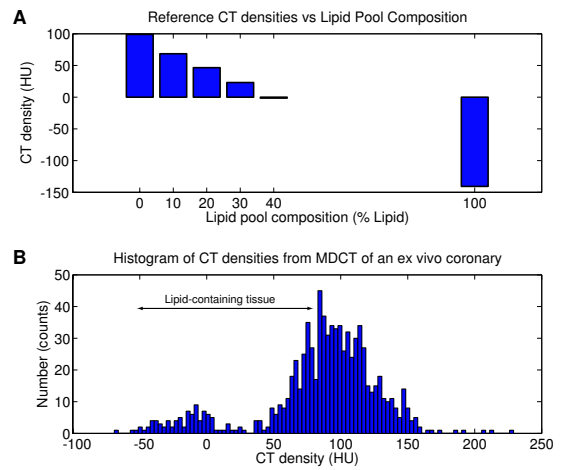

Figure 2. A. Reference CT densities for our lesion phantoms and B. CT density histogram from ex vivo coronaries.

Figure 2A shows reference CT densities measured from our 6 lesion compositions (\%lipid/\%fibrous volume ratios of $0 / 100,10 / 90,20 / 80,30 / 70,40 / 60,100 / 0)$. Comparison of these values with a histogram of CT densities from ex vivo coronary plaque containing lipid-rich and fibrous tissue (Figure 2B) indicates that the CT densities of our emulsions fall within the range associated with lipid-containing tissue. The CT density of fibrous tissue was simulated with a solution consisting of $57 \mu \mathrm{L}$ of iodinated contrast (Ultravist$370^{\circledR}$, Schering, Berlin, Germany) in $10 \mathrm{~mL}$ of water. This solution was emulsified with vegetable oil in varying proportion to produce the lesion compositions of interest.

\section{Imaging}

Vessels and lesions were placed into an anthropomorphic
Cardio CT thorax phantom (Quality Assurance in Radiology and Medicine, Moehrendorf, Germany) and scanned on a Siemens Somatom Sensation 16 Cardiac MDCT scanner (Siemens Medical Solutions, Forcheim, Germany). A cardiac spiral imaging protocol was used $(80 \mathrm{kVp}, 500 \mathrm{mAs}, 16 \times 0.75 \mathrm{~mm}$ collimation, $0.75 \mathrm{~s}$ rotation time, $6 \mathrm{~mm} / \mathrm{s}$ table feed, $22 \mathrm{~cm}$ field-of-view, and a $512 \times 512$ pixel matrix) together with an ECG simulator which provided a $60 \mathrm{bpm}$ trigger for image reconstruction. Images were reconstructed with $0.75 \mathrm{~mm}$ thick slices and a medium smooth image reconstruction filter (B35F). IVUS (Boston Scientific, Boston, MA) imaging with linear pullback of a $40 \mathrm{MHz}$ probe was used as a reference method for validation of MDCT-based vessel morphology measurements. Vessels were then sectioned at regular intervals and photographed for use as a histology-equivalent reference.
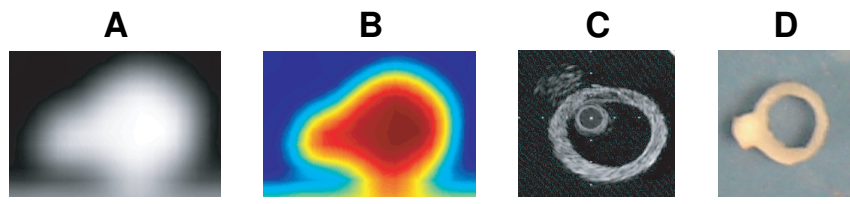

Figure 3. Vessel morphology images showing A. an MDCT cross-section in grayscale, B. the same MDCT image in false color, $\mathbf{C}$. the corresponding IVUS image, and D. the corresponding "gross section".

\section{Analysis}

Analysis of morphology was performed in MATLAB (MathWorks, Natick, MA) on corresponding images from MDCT, IVUS, and digitized gross section data from 44 cross-sections. MDCT images were visualized with a false color map in order to facilitate detection of the lumen boundary as indicated in Figure 3. Two independent experts without prior knowledge about phantom characteristics manually segmented the MDCT images. Detected vessel boundaries at the inner and outer walls were used to compute morphological parameters of vessel area and equivalent vessel diameter ${ }^{1}$. IVUS and digitized gross section data were manually segmented by a separate reader who was blinded to the MDCT results. Measurements of vessel morphology between modalities and between readers were compared with Bland-Altman plots.

For the analysis of lipid pool detection, MDCT images of the lesion phantoms in cross-section were cropped down to $20 \times 20$ pixel patches that may or may not have included a lipid pool. These subimages were presented in random order to 2 independent readers who annotated whether a lipid-rich region was present within the fibrous tissue. The boundaries of any detected lipid pool were also identified to estimate size and CT density. Detection sensitivity and specificity were then derived as a function of lipid pool size

\footnotetext{
${ }^{1}$ defined as the diameter of a circle with the same vessel area
} 
and composition. Finally, the accuracy and variability of lipid pool measurements was examined with Bland-Altman analysis.

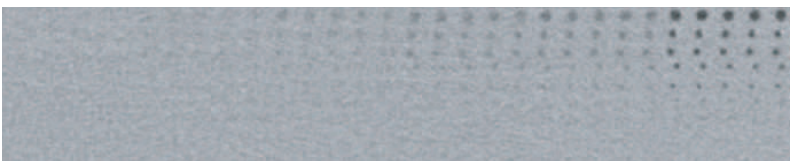

Figure 4. All 350 lesion subimages shown in a single montage. Lipid pool concentration increases from left to right and diameter decreases from top to bottom. Five samples for each lipid pool size and composition were evaluated.

\section{Results}

Figure 5 shows Bland-Altman plots comparing MDCT and IVUS measurements of vessel diameters and areas. These plots show the measurement difference between MDCT and IVUS as a function of the averaged measurements. The measurement bias is indicated by a solid horizontal line and $95 \%$ confidence intervals are indicated by dotted horizontal lines. Diameter and area measurement biases for MDCT relative to IVUS were $0.03 \mathrm{~mm}$ and $-0.57 \mathrm{~mm}^{2}$ respectively, indicating accurate morphology estimation on average. The standard deviation of measurement errors was $0.60 \mathrm{~mm}$ for equivalent diameters and that for vessel areas was $3.32 \mathrm{~mm}^{2}$.
A

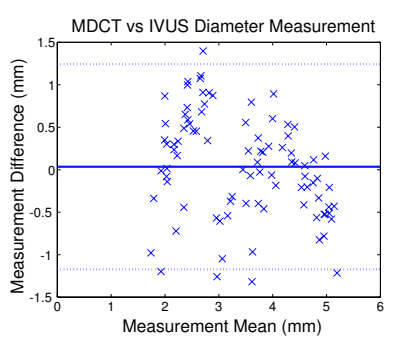

A

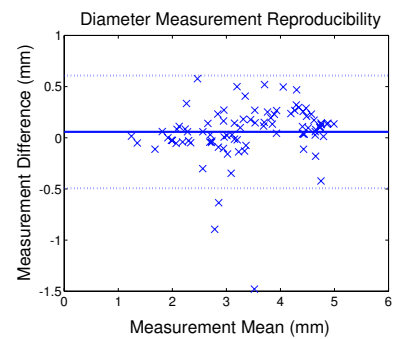

B

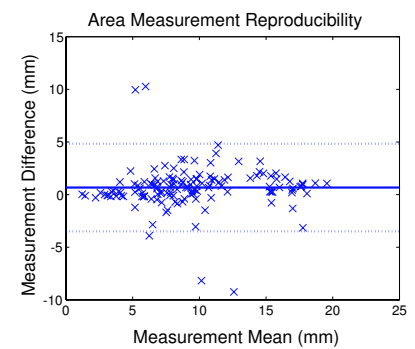

Figure 6. Interobserver Bland-Altman plots comparing A. diameters, and B. areas.

and $0.54 \mathrm{~mm}$. For area measurements, the bias and standard deviation were $1.44 \mathrm{~mm}^{2}$ and $3.32 \mathrm{~mm}^{2}$. The slight overestimation of dimensions from MDCT relative to gross sectioning may be due to PVA hydrogel shrinkage during the interval between imaging and sectioning.

Figure 6 shows the interobserver reproducibility of MDCT-based morphology measurements. The agreement between observers is higher than between modalities, as demonstrated by the tighter clustering of measurement errors. The interobserver error bias and standard deviation for equivalent diameters were $0.06 \mathrm{~mm}$ and $0.28 \mathrm{~mm}$, whereas for vessel areas, they were $0.67 \mathrm{~mm}^{2}$ and $2.08 \mathrm{~mm}^{2}$.

Lesion detection sensitivity and specificity were similar between Readers 1 and 2 . Overall, the specificity of lesion assessment was $96 \%$, and the detection sensitivity for any lipid-containing lesions between $0.4-4.0 \mathrm{~mm}$ in diameter was only $37 \%$. Considering only lipid pools between $1.0-4.0 \mathrm{~mm}$ in diameter, the sensitivity rose to $54 \%$ and for cores between $1.5-4.0 \mathrm{~mm}$ in size, the detection sensitivity was $63 \%$. Figure 7A illustrates the change in detection sensitivity for cores $\geq 1.5 \mathrm{~mm}$ containing lipid concentrations $\geq 10 \%, \geq 20 \%, \geq 30 \%, \geq 40 \%$, and $100 \%$. An $88 \%$ detection sensitivity is possible for cores $\geq 1.5 \mathrm{~mm}$ (or $1.7 \mathrm{~mm}^{2}$ ) containing $\geq 30 \%$ lipid. Figure $7 \mathrm{~B}$ shows the variation in detection sensitivity for all cores with $\geq 30 \%$ lipid as a function of diameter range.

Figures 8A-B demonstrate the quality of lipid-pool diameter and CT density estimates from MDCT. Overall, diameter estimation bias and standard deviation were $0.34 \mathrm{~mm}$ and $0.79 \mathrm{~mm}$ respectively (area bias and standard deviation were $1.34 \mathrm{~mm}^{2}$ and $3.49 \mathrm{~mm}^{2}$ ). These values are somewhat worse than those from vessel morphology measurement, reflecting the difficulty of measuring necrotic cores with MDCT. The plot of CT density estimation errors indicates that there is substantial variability in $\mathrm{HU}$ measurements for a given lesion composition. Furthermore, as the \%lipid concentration increases, there is an increasing overestimation of CT density. This observation may be explained by the fact that CT densities depend not only on \%lipid concentration, but also on the lipid pool size due 
A

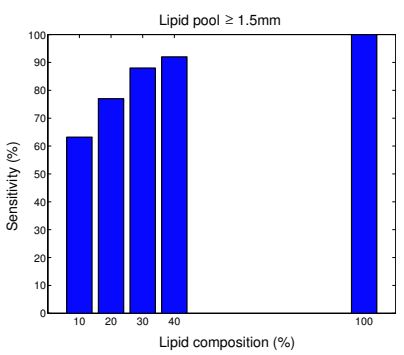

B

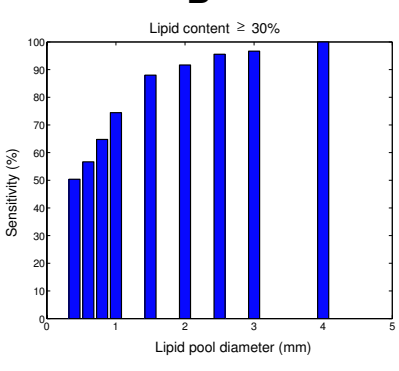

Figure 7. Detection sensitivity as a function of A. lesion composition for diameters between $1.5 \mathrm{~mm}$ and $4.0 \mathrm{~mm}$, and B. lipid pool diameter for lesion compositions with $\geq 30 \%$ lipid.

to partial voluming (Figure 8C). The estimation of \%lipid content in a given lipid pool based on measured CT density will therefore be difficult because of measurement noise and blurring.

\section{Conclusions}

We have characterized the performance of 16-slice MDCT for vessel morphology measurements and lipid pool assessment. Morphology measurements with MDCT are accurate relative to reference imaging, with good interobserver reproducibility. Detection of lipid-containing regions is possible with sensitivities $\geq 88 \%$ and specificity of $96 \%$ for lesion compositions with $\geq 30 \%$ lipid and $\geq 1.5 \mathrm{~mm}$ in size. The estimation of lipid pool dimension from MDCT is more difficult than vessel morphology measurement and measured CT densities within a lesion

A
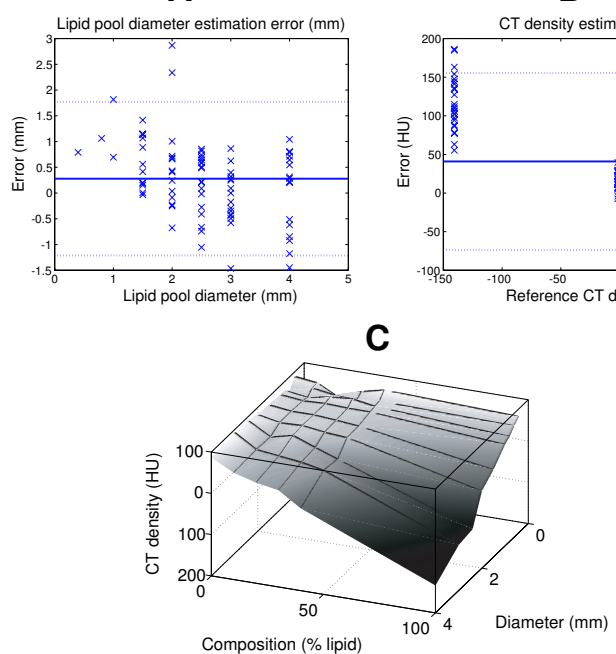

Figure 8. Estimation error in A. lipid pool size, and B. CT density as a function of diameter. C. CT density (HU) as a function of lipid pool diameter and \%lipid composition. cannot be used directly to determine the \%lipid content due to noise and limited spatial resolution. The results described reflect the optimal performance characteristics of 16-slice MDCT since they were obtained from phantombased experiments conducted in the absence of cardiac and respiratory motion. Despite these limitations, our findings are useful since they constrain our interpretation of MDCT data obtained in vivo. We anticipate that the level of measurement quality and lesion detectability shown here will improve as we develop more sophisticated methods for MDCT image reconstruction, processing, and analysis.

\section{Acknowledgements}

This research was supported in part by the NIH Radiological Science Training Grant 5-T32 Ca 09362 E 20, the Center for the Integration of Medicine and Innovative Technology (CIMIT, Partners Healthcare Systems), the New York Cardiac Center, and the Deutsche Forschungsgemeinschaft, Bonn, Germany.

\section{References}

[1] Virmani R, A.P. B, Kolodgie F, Farb A. Vulnerable plaque: the pathology of unstable coronary lesions. Journal of Interventional Cardiology 2002;15(6):439-446.

[2] Ge J, Chirillo F, Schwedtmann J, Görge G, Haude M, Baumgart D, Shah V, von Birgelen C, Sack S, Boudoulas $\mathrm{H}$, Erbel R. Screening of ruptured plaques in patients with coronary artery disease by intravascular ultrasound. Heart 1999;81:621-627.

[3] Nieman K, Cademartiri F, Lemos P, Raaijmakers R, Pattynama P, de Feyter P. Reliable noninvasive coronary angiography with fast submillimeter multislice spiral computed tomography. Circulation 2002;106:2051-2054.

[4] Ropers D, Baum U, Pohle K, et al. Detection of coronary artery stenoses with thin-slice multi-detector row spiral computed tomography and multiplanar reconstruction. Circulation 2003;107:664-666.

[5] Schroeder S, Kopp A, Baumbach A, Meisner M, Kuettner A, Georg C, Ohnesorge B, Herdeg C, Claussen C, Karsch $\mathrm{K}$. Noninvasive detection and evaluation of atherosclerotic coronary plaques with multislice computed tomography. Journal of the American College of Cardiology 2001; 37:1430-1435.

[6] Becker C, Nikolaou K, Muders M, Babaryka G, Crispin A, Schoepf U, Loehrs U, Reiser M. Ex vivo coronary atherosclerotic plaque characterization with multi-detectorrow ct. European Radiology 2003;13:2094-2098.

Address for correspondence:

${ }^{*}$ Raymond C. Chan and * Maros Ferencik

Department of Radiology, Massachusetts General Hospital 100 Charles River Plaza, Suite 400 Boston, MA 02114 USA. rchan@nmr.mgh.harvard.edu, maros_ferencik@hms.harvard.edu 